\section{AIAA-2003-2708 LOCKHEED MARTIN RESPONSE TO THE OSP CHALLENGE}

\author{
Robert T Sullivan", Randy Munkres ${ }^{\dagger}$, Thomas D \\ Megna $^{\ddagger}$, and Joanne Beckham ${ }^{\S}$ \\ Lockheed Martin, Denver, Colorado
}

\begin{abstract}
The Lockheed Martin Orbital Space Plane System provides crew transfer and rescue for the International Space Station more safely and affordably than current human space transportation systems. Through planned upgrades and spiral development, it is also capable of satisfying the Nation's evolving space transportation requirements and enabling the national vision for human space flight. The OSP System, formulated through rigorous requirements definition and decomposition, consists of spacecraft and launch vehicle flight elements, ground processing facilities and existing transportation, launch complex, range, mission control, weather, navigation, communication and tracking infrastructure. The concept of operations, including procurement, mission planning, launch preparation, launch and mission operations and vehicle maintenance, repair and turnaround, is structured to maximize flexibility and mission availability and minimize program life cycle cost. The approach to human rating and crew safety utilizes simplicity, performance margin, redundancy, abort modes and escape modes to mitigate credible hazards that cannot be designed out of the system.
\end{abstract}

\section{THE ROLE OF THE ORBITAL SPACE PLANE} PROGRAM IN THE NATIONAL, HUMAN SPACE FLIGHT VISION

Space continues to capture the imagination of governments, industry and all humanity. It holds the promise of boundless scientific discovery, exploration, economic expansion and military dominance. At this moment an orbiting laboratory is awaiting completion and research operations, the next generation space

This work is unclassified and publicly available. It was performed under Contract No. NAS8-01098 as part of NASA's Orbital Space Plane Program. Presented at the International Air \& Space Symposium and Exposition, Dayton, Ohio 14-17 July 2003.

AIAA Member, OSP Spacecraft System Design Lead

${ }^{\dagger}$ AIAA Member, OSP Operations Manager

¥ AIAA Member, OSP Spacecraft Manager

$\S$ AIAA Member, OSP Deputy Program Manager telescope is in design, crewed missions to the Moon and Mars are being planned and entrepreneurs are vying for a future space tourism market. The cornerstone of these missions and those not yet conceived is safe, reliable, affordable transportation between terra firma and low earth orbit. NASA's Orbital Space Plane (OSP) program, intended initially to service the International Space Station (ISS) more quickly, more safely and more affordably than current space transportation systems, is also the next step to the goal of routine space access. Lockheed Martin's modular OSP System satisfies the immediate need for crew rescue and transfer for ISS and, through planned upgrades and strategic spiral development, provides a growth path for future systems that accommodate the Nation's evolving space transportation needs, enabling the realization of the national human space flight vision.

\section{OSP SYSTEM REOUIREMENTS AND FUNCTIONALITY}

Designed to transfer up to four (4) crew to and from low earth orbit, the Lockheed Martin OSP System enables space station complete operations as soon as practical by assuring safe, reliable and affordable human access to ISS and providing crew rescue for the US segment crew complement. The System is also capable of delivering limited contingency cargo in lieu of crew. The OSP will be significantly safer than current space transportation systems, managing risk through hazard elimination and mitigation, performance margin, redundancy and crew escape. Designed for simplicity, autonomous operations, robustness and operability the OSP system will require less time and resources to design, prepare for and execute a mission, minimizing training and operations costs and thus life cycle cost. The Lockheed Martin OSP System is composed of spacecraft and launch vehicle flight segments, ground processing and launch facilities and existing communication and navigation infrastructure that maximize current mission flexibility and supplies the building blocks for accommodating evolved missions at an affordable price. Lockheed Martin has embraced the government's System Design Philosophy, implementing rigorous System Engineering processes to decompose and mature functional requirements, minimize interfaces and optimize the entire system. 


\section{OSP SYSTEM DEFINITION}

The OSP System includes all of the elements required to accomplish the crew transfer and rescue missions and that contribute to the lifecycle cost of those missions from the moment it is an idea until it is retired. The elements can be categorized as heritage systems (ISS, ELV, eastern range, TDRSS, etc.), physical objects (spacecraft, manufacturing facilities, software, flight simulators, thermal vacuum test chamber, ground support equipment, commodities, shipping containers, etc.), human resources (managers, mission analysts, technicians, information technology support, skills instructors, grounds maintenance crews, etc.), processes (engineering releases processes, nonconforming material control, component refurbishment process, etc.) and information (I-loads, aerodynamics database, component historical performance, etc.). The summary of the OSP System is depicted in Figure 1.

The OSP Program boundary delineates between the OSP unique system elements that must be designed and acquired by the OSP Program and existing elements or stand-alone systems that are utilized to accomplish the OSP mission.

\section{International Space Station Program}

The ISS Program is the primary OSP System customer and the source for key functionality and interface requirements. The ISS configuration is assumed to be Core Complete with Node 3 . The ISS Program will plan the visiting vehicle traffic model, OSP mission manifest and mated operations. During proximity operations the ISS crew will monitor the approach and departure and have abort command capability. While mated, the ISS will provide communication, navigation, power, thermal control and atmospheric revitalization services to the OSP. The Space Station Processing Facility (SSPF) will process ISS payload items and all flight crew equipment such as suits, mission related tools and personal provisions.

\section{OSP Spacecraft. Ground Processing Facility} and Transportation

The OSP spacecraft, composed of both reusable and expendable elements, contains all the crew escape, thermal protection, mechanisms, propulsion, flight control, navigation, communication, power, atmospheric pressurization and revitalization, thermal control and crew subsystems required to support the crew and perform nominal and contingency transfer and

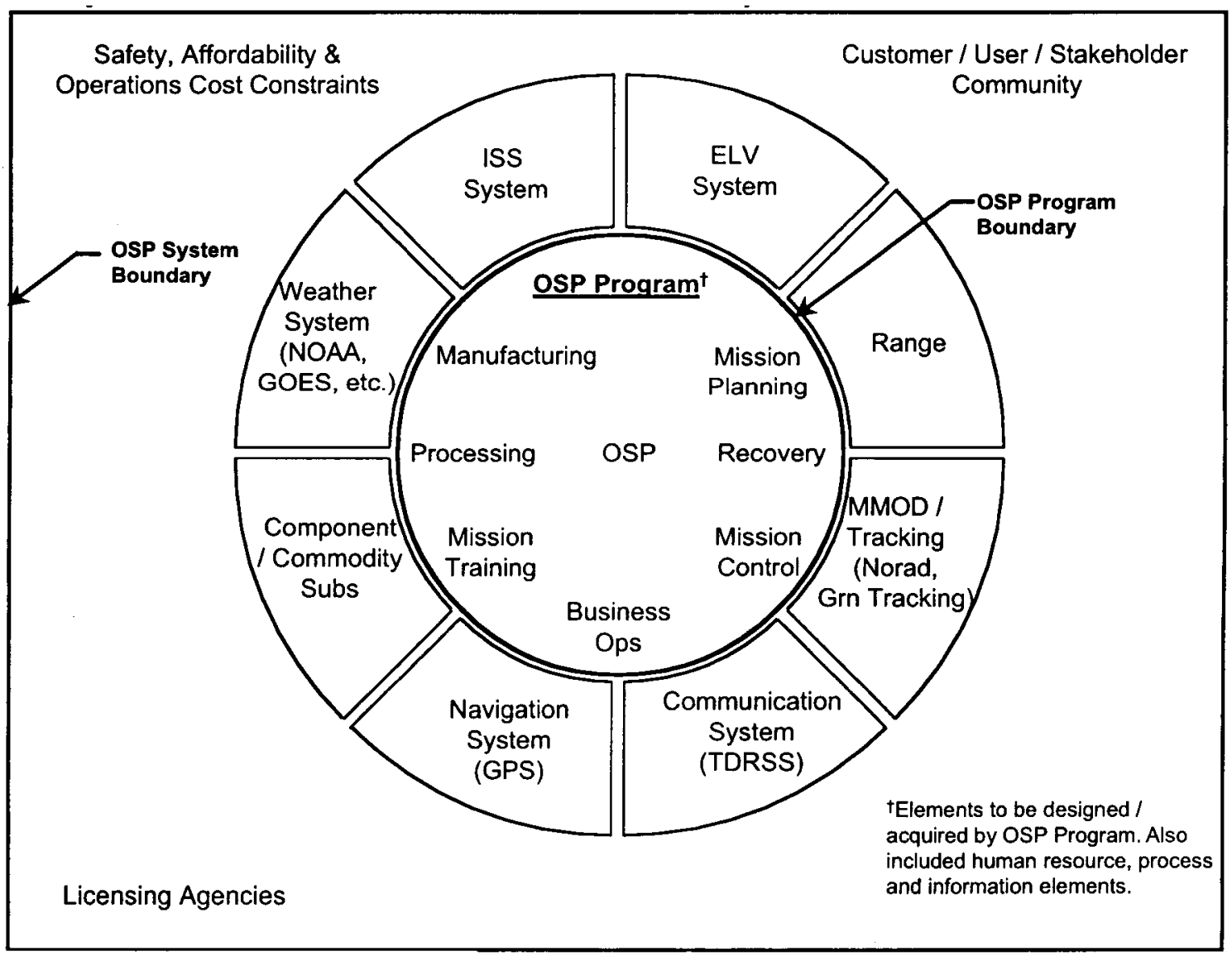

Figure 1 OSP System Elements 
rescue flight operations.

The Space Port Florida Reusable Launch Vehicle (SPFRLV) hangar at KSC is the baseline processing facility for the OSP spacecraft. It is of adequate size to support turnaround processing, planned maintenance and overhaul for multiple vehicles. The processing floor measures $185^{\prime} \mathrm{w} \times 200$ 'd with the average height of $30^{\prime}$. The maximum height at the center is $60^{\prime}$. Presently it has no major services in place except lighting and HVAC. There are stubs for power and communications. Additional personnel and support facilities are required to process the OSP fleet. This hangar is conveniently located on the Shuttle Orbiter tow way near the south end of the $15,000 \mathrm{ft}$. runway of the Shuttle Landing Facility (SLF) and can receive the major OSP flight elements for checkout, integration and servicing. The RLV hangar will contain all the necessary handling GSE, work stands, local control checkout equipment, power and communications interface equipment and the allowed servicing equipment to support turnaround processing. Additional heritage facilities such as the Payload Hazardous Servicing Facility and the Parachute Refurbishment Facility and ground support equipment such OMS/RCS servicing system, portable purge units, portable PRSD/fuel cell service cart and portable power units will be utilized as required.

All flight elements of the OSP are transported from the factory and recovery sites to the SLF via C5 or $\mathrm{C} 17$ aircraft. Transport cradle and dollies serve as portable work stands for each element and allow for elevation alignment and rotation to the vertical for servicing. Once servicing is complete the dollies facilitate integration of the OSP elements. After integration a strongback assembly is attached along the sides of the completed OSP assembly and hoisted onto the transport trailer, where is held securely in place by trunnions for transport to the launch vehicle integration facility.

\section{Launch Services}

The OSP spacecraft will initially be launched on existing medium and heavy lift expendable launch systems. The Government will procure launch services from one or more domestic launch service providers.

The launch service providers, Lockheed Martin and Boeing, will perform launch mission analysis, integrate the OSP to the launch vehicle, perform system tests, conduct launch readiness reviews, conduct launch pad operations and perform countdown and launch procedures. They will monitor launch vehicle performance and record telemetry for post flight analysis.

The OSP program goal is to minimize impacts to the commercial launch system and to capitalize on the financial benefits that come from leveraging the commercial infrastructure. However, some vehicle, process and facility modifications are required for human flight. Examples include the addition of IVHM and crew escape sensors to the launch vehicle, flight safety reviews to preflight operations and a crew access tower to the launch complex.

\section{Launch Range}

The OSP System will utilize the existing infrastructure and personnel at the US Air Force Eastern Range.

\section{Business Operations. Mission Planning \& Training}

The OSP System will utilize existing business operations and mission planning organizations, such as the Astronaut and Flight Surgeon Offices and the Flight Dynamics Branch at the Johnson Space Center in Houston. Many exist training facilities such as the Neutral Buoyancy Laboratory, Remote Manipulator Arm Training Facility, International Space Station Simulator and Hypobaric Chamber will be used for astronaut skills and proficiency training. In addition, new, OSP unique facilities, such as the OSP Single Systems Simulators, OSP Cockpit and Crew Habitat Simulator and Space Operations Simulator will be constructed.

\section{Mission Control}

OSP mission control will be coordinated from the Mission Control and ISS Mission Control Centers located at the Johnson Space Center in Houston.

\section{Nominal, Rescue and Escape Recovery Infrastructure}

Recovery and turnaround facilities consist of nominal and emergency landing site locations and facilities supporting vehicle maintenance and turnaround operations.

The OSP primary and secondary landing sites support all phases of final tracking, approach and landing operations. The landing sites provide personnel, support facilities, OSP handling and transport equipment, safing \& de-servicing GSE, portable power, environmental purge, communications, landing aids and fire/rescue and medical support.

Sea. air and land based crew recovery teams will be stationed strategically at existing coast guard and military installations along the ascent flight path.

\section{Weather, Navigation, Tracking, Communication and MMOD Systems}

Heritage National Oceanic and Atmospheric Administration ground and space based weather sensing infrastructure and the National Weather Service forecast service will provide current and outlook weather forecasts at the launch facility, along the ascent and decent flight paths and at the primary and 
secondary landing sites. In addition, the Eastern Range will collect day of launch wind data.

The Global Positioning System (GPS) will provide navigational data to the OSP while in orbital free flight. Precise navigation during ISS docking/ berthing and landing will be accomplished with differential GPS, radar, lidar and radio navigational aids. Both existing ground and new space based options are being considered for ascent range tracking.

Communication and telemetry will be routed between the OSP and the Mission Control Center via and S-band RF link with the existing NASCOM and TDRSS infrastructure. In addition, UHF is available for communication between the OSP spacecraft and ISS while in close proximity.

The North American Aerospace Defense Command will perform micrometeoroid and orbital debris tracking and orbit propagation.

\section{Ground Operations}

\section{OSP OPERATIONS}

\section{Procurement}

Constraining OSP integration operations to existing expendable launch vehicle processes and infrastructure result in a nominal order to launch time of 9-15 months. This time spans from mission procurement through launch. However, the order to launch process may be minimized through implementation of operational strategies that include launch vehicle block buy, a dedicated OSP integration facility or maintaining a flight ready OSP and launch vehicle stack.

\section{Mission Planning and Analysis}

Once the OSP mission is identified and procurement begun, detailed mission unique planning and analysis is performed.

To the greatest degree practical, the OSP System will utilize performance margin, class analysis, mission flexibility and robustness to minimize the required mission unique analysis.

\section{Recovery. Turnaround and Cargo Operations}

OSP ground and cargo operations consist of two types of operations; standard OSP and cargo turnaround maintenance and unplanned maintenance operations. The standard OSP mission turnaround begins at vehicle touchdown where safing and recovery operations begin at the landing site. Once the flight crew egresses the vehicle, the ground crew completes safing operations and prepares the vehicle for transport to the SPFRLV Hanger. With the vehicle in the processing facility in its support stand/transport trailer, planned and unplanned maintenance operations are performed on the OSP. This includes subsystem deservicing where required by safety, removal of expendable hardware, consumable replacement, inspection of the thermal control System (TPS) and satisfaction of rolling maintenance requirements, such as batteries and pyrotechnic removal \& replacement. Any unplanned maintenance that the IVHM system has identified is scheduled to fit seamlessly into the standard turnaround maintenance plan and the appropriate work authorizing documents are produced and released to the processing area for work. When cargo is flown in lieu of flight crew any down cargo or cargo accommodations hardware is removed and returned to the SSPF for customer disposition.

Once all vehicle subsystem planned and unplanned maintenance work is complete early prelaunch stowage and servicing operations commence. Allowable hazardous servicing operations are performed in the RLV hangar, such as battery installation and high pressure gas servicing, and those that can't be performed there are performed in the HPSF or other NASA approved facility. Once servicing of storable commodities is complete, for example hypergolic propellant, high pressure GN2 \& GHe, and potable water, etc., the vehicle undergoes final integration in the RLV hangar where the escape systems and launch vehicle adapter are installed. With the OSP in configuration for launch vehicle integration, final stowage of crew related equipment and planned cargo items are accomplished.

With final closeouts and rollout readiness verified the vehicle is rolled out to the launch complex for integrated operations that include erection, stacking and final checkout and servicing.

\section{Integration and Launch Operations}

Integration operations for the OSP start when the standalone operations have been completed in the OSP hangar and the vehicle is ready for integration to its launch vehicle. The OSP design enables it to be launched on either an Atlas V from launch complex 41 or the Delta IV from launch complex 37.

Integration at the launch sites can start when the launch vehicle is assembled and the Launch Vehicle Readiness Tests (LVRT) are successfully completed. These tests verify that the launch vehicle ground and airborne systems are compatible and capable of proper integrated system operation. The launch vehicle is then prepared to accept the OSP.

OSP mating to the launch vehicle occurs about one week prior to launch. After arrival at the launch complex, the OSP is positioned for hoisting onto the launch vehicle. Environmental conditioning is transferred from the portable unit used during transport to the launch complex source and is available during hoisting operations. A hoisting sling is fastened to the OSP assembly-lifting fixture and tie-downs to the OSP ground transport vehicle are released. The OSP is then 
lifted onto the launch vehicle. After mechanical attachment of the OSP to the launch vehicle, the lifting fixture is removed. Electrical connections between the OSP and the launch vehicle and the OSP and ground are mated to complete the operation.

The inter-vehicle connections include, power, command and communications.

There are two OSP to ground umbilicals, a pre-launch and T-0. The pre-launch umbilical, utilized for fuel cell loading operations, is fully automated capability of retracting prior to $\mathrm{T}-0$ and re-mate in the event of a launch abort.

The T-0 umbilical, used to supply ATCS cooling and environmental purge, is retracted upon launch commit.

OSP operations after integration to the launch vehicle involve final installations of ordnance, escape module final preparations and late cargo stowage. Late cargo stowage will occur prior to the start of launch operations and start no later than T-16 hours.

Prior to entering launch operation final ordnance installation and checkout is completed all GSE removed. An Integrated Vehicle Test (IVT) will be performed to provide a launch readiness verification of the OSP, launch vehicle, ground and airborne systems.

The launch operations timeline is similar to a commercial launch. However, unique operations such as OSP fuels cell reacting loading and crew ingress will be inserted.

Fuel cell reactant loading occurs within the existing launch vehicle loading operations. The ingress of the crew will occur after the propellant loading has transitioned into a replenish phase.

\section{Nominal Crew Transfer and Rescue Vehicle Exchange Flight Operations}

The flight operations profile for nominal transfer and rescue vehicle exchange missions are similar. Both can be a flown with any complement of crew and cargo.

\section{Terminal Count Down}

Terminal launch countdown begins once the closeout crew has cleared the pad. The access platform is retracted and the OSP and the launch vehicle execute automated system checks and are configured for launch. The range, launch and mission control teams verify launch readiness status.

\section{Launch}

At launch commit, the launch conductor imitates the booster engine auto start sequence. Once onboard sensors indicate the engines have achieved full thrust, the launch vehicle propellant and power umbilicals are retracted and the hold down mechanism is released. The launch vehicle guidance flies a preprogrammed ascent trajectory, adjusting for off nominal wind conditions, while the OSP and mission control passively monitor launch vehicle performance, attitude, rates and system status. Upon exhaustion of booster propellants, the booster engines shut down, the booster separates from the stack and the upper stage engine ignites. Once upper stage engine nominal steady state operation is confirmed the launch escape solid rocket motors are jettisoned. The upper stage closed loop guidance then flies the OSP to the planned low earth orbit insertion point, performing multiple burns and coasts as required. After upper stage engine cutoff the upper stage holds its attitude while the OSP initiates spacecraft separation and performs a collision avoidance maneuver.

\section{Orbit Transfer and Phasing}

When clear of the upper stage the spacecraft performs orbit transfer and ISS phasing maneuvers. The OSP subsystems are sized such that power and thermal control maneuvers are not required. At the same time the upper stage performs a disposal burn, reenters and breaks up over an ocean. At a convenient point in the mission timeline, the crew can doff their launch entry suits, eat and rest.

\section{Operations}

ISS Rendezvous and Arrival Proximity

Within 2 days of launch, the OSP rendezvous with the ISS outside the Approach Ellipsoid (AE), such that a free drift trajectory will not intercept the ISS trajectory in fewer than 24 hours. Upon receiving authorization from ISS crew, the OSP enters the AE, intercepting the preprogrammed approach corridor centered on the docking/berthing port outside the Keep Out Sphere (KOS). The OSP then either docks to or is captured by the ISS Mobile Servicing System (ISSMSS) and berthed to ISS. Both the arriving and departing OSP will remain mated to ISS while the crew rotation and cargo exchange is completed.

\section{Mated Operations}

After hard dock is established, the access tunnel is pressurized and a leak check is performed, the OSP and ISS hatches are opened. Communication, telemetry, power, thermal control coolant loop and atmosphere revitalization umbilicals are then connected. Non-critical avionics, power and lifesupport systems are shut down and the OSP is configured in mated standby mode. OSP can remain quiescent mode for up to 180 days or until required for crew exchange or rescue from ISS. 


\section{Crew \& Cargo Exchange}

ISS cargo and crew personal effects are transferred from the OSP and stored on Space Station. In addition, scientific or high value cargo that is to accompany the ISS crew completing their deployment to earth is stowed on board the departing OSP. ISS change of command and experiment responsibility handoff occurs following a status briefing. Periodically, while attached to ISS, the OSP is powered up temporarily for system diagnostic and checkout.

\section{OSP De-mate. Departure Proximity \\ Operations and Deorbit}

Upon the decision to depart ISS the crew don their launch entry suits, ingress the OSP, shut the ISS and OSP hatches and perform hatch leak checks. The OSP crew then powers up the dormant systems, uploads final flight data package and landing site weather data, establishes navigational state and communication links, configures vehicle for departure and performs system health checks. When ready, the access tunnel is depressurized, the OSP separates and exits the approach ellipsoid. Prior to reentry, while awaiting the alignment of the spacecraft ground track with the landing site, the crew conducts thermal control procedures to cold soak the radiators and thermal protection system. At the start of the reentry window, a direct deorbit burn is initiated.

\section{Landing and Recovery}

Upon entry interface the OSP performs a reentry and is recovered at the primary landing site within 24 hours of separation from ISS. Once the OSP is safed by the recovery team, the deconditioned crew egress the vehicle via the access hatch and are attended to by recovery team physicians. Time critical payload is removed and transported to the SSPF for post flight processing and returned to the payload principle investigator. The OSP is hoisted onto the transport fixture and trucked back to the SPFRLV hanger for refurbishment.

\section{Rescue, Abort and Escape Flight Operations}

In general, the OSP system design philosophy of hazard elimination, performance margin, redundancy and graceful degradation mitigates the consequences of system failures. However, abort and escape options are provided for all flight regimes that have credible failure modes for which recovery is not possible.

\section{Launch Pad Abort}

In the event that a hazardous condition arises at the launch complex prior to launch commit, OSP crew and launch vehicle closeout crew can expeditiously evacuate the area via the launch complex emergency evacuation system. The launch vehicle and OSP are safed from the launch control center.

\section{Launch Pad Escape}

If the hazard arises after crew cabin closeout and the crew cannot exit the spacecraft safety a pad escape is commanded by the crew, mission control, launch control or the automated escape initiation system. The separation mechanism ensures positive clearance between the OSP and launch vehicle. After escape motor burn out and coast, parachutes and impact attenuation airbags deploy. Additionally, if wind conditions result in a water landing, float bags are inflated to ensure the OSP remains buoyant and floats upright. Fire and rescue crews respond immediately to recover the crew. It is understood that execution of a launch pad escape may result in the destruction of the launch vehicle and launch complex.

\section{Ascent Escape}

If the launch vehicle does not impart enough energy for the OSP to achieve orbit or ascent abort, or in the event a catastrophic launch vehicle structural failure or loss of control an ascent escape is commanded by the crew, mission control, launch control or the automated escape initiation system. The event timeline for ascent escape, initiated during boost phase, is similar to a launch pad escape. However, prior to separation the OSP commands thrust termination. Also, dependent on OSP state at escape initiation, exoatmospheric coast and atmospheric reentry occur prior to drogue and main chute deployment. OSP escape instantaneous impact point crosses the Atlantic Ocean, Europe, the Arabian Peninsula and the Indian Ocean. The nearest sea, air or land based recovery team is deployed to recover the crew. Survival equipment is provided for the crew until the recovery team arrives.

\section{ISS Approach Abort}

While inside the ISS AE, the OSP provides system status and relative state data to the ISS. OSP crew, space station crew and mission control crew monitors the approach and has the capability to command an abort. Collision Avoidance Maneuvers (CAM) initiated within the KOS will begin with by establishing a positive opening rate and a CAM initiated within the $A E$ will exit the $A E$ in fewer than 90 minutes. If an approach abort is executed the OSP moves to a predetermined safe hold point to await anomaly resolution and approach resumption. The OSP is capable of maintaining a contingency hold for up to 34 hours. The OSP is capable of two approach attempts. After the second failure the OSP departs ISS and returns to the nominal landing site.

\section{ISS Crew Rescue}

There are two OSP crew rescue operational scenarios. The first requires immediate departure from 
the ISS, while the second requires timely, but not emergent return to earth. Example events that could precipitate an immediate evacuation of ISS include catastrophic station depressurization, atmospheric contamination, fire or loss of control. In these scenarios the ISS crew quickly enters the OSP and isolates its environment from that of the ISS. An abbreviated power up and departure checklist is performed and the OSP the separates from the ISS and maneuvers to a safe position. While the ISS Mission Control Center attempts to reestablish a safe environment on ISS, the OSP crew can complete the departure sequence, system health checks, verify landing site availability and perform landing site phasing. If space station control is reestablished then the OSP can return the crew to ISS. Otherwise, the space station crew can abandon ISS and return to earth at the primary landing site or one of the contingency landing sites.

Example events that require timely departure from ISS include crew illness or non-catastrophic, but not recoverable ISS system failure. Crew rescue from ISS system failures imply that the OSP must be able to perform the separation and departure without support from the International Space Station, including navigational state information. In these scenarios, the departing ISS personnel don their launch injury suits, ingress the OSP and begin the departure checklist. The OSP phases with the landing site while mated to ISS, initiating separation as dictated by the return mission time line, included in the flight data package upload.

Consideration of contingency landing sites at Edwards, Minot and Malstrom Air Force bases in addition to the primary landing site in Avon Park, Florida assure crew rescue can be accomplished in significantly less than the required 24 hours.

\section{Landing Site Weather Contingency}

The Lockheed Martin OSP spacecraft includes additional contingency power and atmospheric revitalization consumables. The mission duration margin enables more than one opportunity for landing at the primary recovery site to allow for unexpected weather changes before requiring the OSP return to a contingency landing site.

\section{CONCLUSIONS}

The Lockheed Martin OSP concept of operations and robust, comprehensive system design provide safe, reliable and affordable access to the ISS and builds the space transportation foundation for future human space flight missions. 